\title{
Treatment of Severe Localized Cerebral Vasospasm Following Recurrent Hemorrhage from Middle Cerebral Artery Aneurysm \\ -Case Report-
}

\author{
Kazuo KataOKA, Kazuhide NiIYama, Toshifumi UEJima, \\ Ryotaro KURODA, Masahiko IOKU, Kazuo YAMADA*, \\ Mamoru TANEDA* and Toru HAYAKAWA*
}

\begin{abstract}
Department of Neurosurgery, Kinki University School of Medicine, Osaka-Sayama, Osaka; *Department of Neurosurgery, Osaka University Medical School, Osaka
\end{abstract}

\begin{abstract}
A 52-year-old female presented with localized but severe cerebral vasospasm induced by recurrent aneurysmal subarachnoid hemorrhage. The middle cerebral artery (MCA) aneurysm was clipped and the subarachnoid hematoma evacuated 1 day after recurrent hemorrhage. The cerebral vasospasm, localized in a region near the MCA aneurysm, was reduced by papaverine and nicardipine vasodilating agents delivered via an Ommaya cerebrospinal fluid reservoir placed at craniotomy.
\end{abstract}

Key words: cerebral aneurysm, middle cerebral artery, Ommaya reservoir, subarachnoid hemorrhage, vasospasm

\section{Introduction}

Recent advances in microsurgical techniques and the introduction of early surgery have improved clinical outcomes for patients with aneurysmal subarachnoid hemorrhage (SAH). ${ }^{6)}$ However, early surgery is not possible for all patients, so cerebral vasospasm and rebleeding continue to cause mortality and morbidity.

We describe a patient with localized cerebral vasospasm at the trunk of the middle cerebral artery (MCA) induced by recurrent hemorrhage from a left MCA aneurysm, and discuss the use of an Ommaya cerebrospinal fluid (CSF) reservoir to deliver vasodilating drugs for the relief of vasospasm.

\section{Case Report}

A 52-year-old female was admitted to a local hospital with sudden onset of severe headache. Computed tomographic (CT) scans 3 days after onset showed mild SAH in the left Sylvian fissure. The headache

Received June 26, 1992;

Accepted May 14, 1993 remained moderate until recurrence of severe headache 14 days after the initial onset. She was transferred to our hospital on the day of recurrence.

Neurological examination revealed moderate expressive aphasia and right hemiparesis. CT scans clearly showed SAH predominantly in the left Sylvian fissure (Fig. 1). Cerebral angiograms revealed bilateral aneurysms at the MCA bifurcation, and severe narrowing of the left MCA at the aneurysm site (Fig. 2). Her preoperative condition was grade 4 according to the Hunt and Hess scale. ${ }^{1)}$

The ruptured aneurysm of the left MCA was clipped via a left craniotomy and the associated hematoma evacuated. The hematoma was localized around the aneurysm, and the ipsilateral MCA was severely narrowed in this region. After aneurysm clipping, intraoperative application of papaverine saline solution $(1 \mathrm{mg} / \mathrm{ml})$ successfully reduced vasospasm. An Ommaya CSF reservoir was therefore placed for drug delivery, with the catheter tip placed near the vasospastic MCA trunk and clipped aneurysm.

She developed right hemiplegia and global aphasia following recovery from anesthesia. $4 \mathrm{ml}$ of papaverine saline solution $(1 \mathrm{mg} / \mathrm{ml})$ was injected 


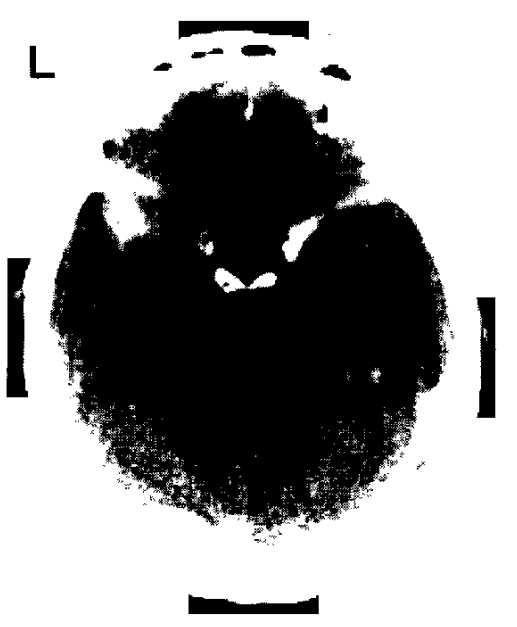

Fig. 1 CT scan 1 day after recurrent hemorrhage, showing left intra-Sylvian hematoma.

over a 2-minute period through the reservoir system. Left carotid angiograms on the day after surgery showed a moderate reduction in the vasospasm compared to that present preoperatively (Fig. 3). A solution of the calcium antagonist nicardipine $(0.2 \mathrm{mg} / 3$ $\mathrm{ml}$ saline) was also delivered via the Ommaya reservoir. Solutions containing papaverine and nicardipine were injected twice a day until $\mathrm{CT}$ scans clearly revealed a low-density area in the territory of the left MCA 2 days after aneurysm surgery. No adverse effects were observed when the drugs were infused. She gradually recovered from the neurological deficits. However, follow-up CT scans revealed cerebral infarction in the territory of the left MCA.

The right MCA aneurysm, which was not associated with hemorrhage, was clipped via a right craniotomy 8 weeks after the first operation. She was discharged home with moderate expressive aphasia and motor weakness of the right upper extremity 3 months after admission. She could walk independently, and care for herself 2 years after onset of SAH.

\section{Discussion}

Cerebral vasospasm usually reaches the maximum intensity 7-14 days after onset of SAH. ${ }^{4)}$ Mechanical stimulation and recurrence of aneurysmal bleeding can increase the severity of vasospasm in the affected arteries. Our patient developed moderate expressive aphasia and right hemiparesis following recurrent hemorrhage 14 days after onset. Preoperative angiography showed that vasospasm was present near the aneurysm, and the preoperative neurological symptoms were attributable to the severe localized

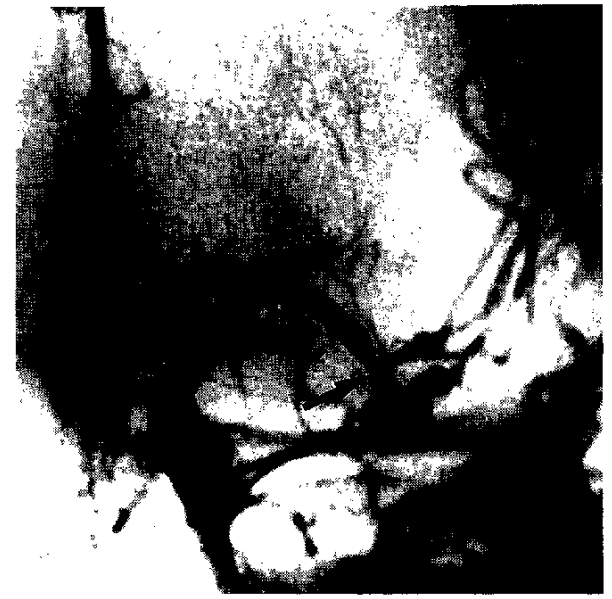

Fig. 2 Left carotid angiogram on the day after recurrent hemorrhage, showing a cerebral aneurysm at the MCA bifurcation and severe arterial narrowing (arrow) near the aneurysm.

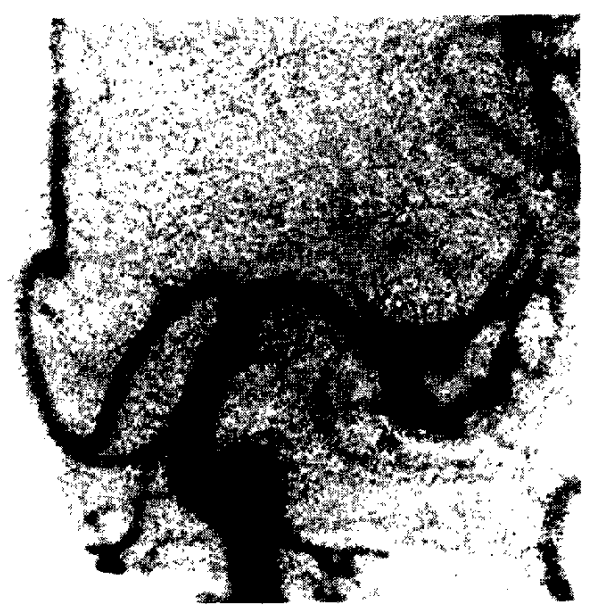

Fig. 3 Left carotid digital subtraction angiogram on the day after clipping of the aneurysm and placement of the Ommaya CSF reservoir, showing disappearance of the aneurysm and moderate increase in the arterial lumen.

vasospasm.

Zabramski et al. ${ }^{7}$ demonstrated that a simulated late rebleeding episode produced intense vasospasm in the "multiple-hemorrhage" canine model. Vasospasm associated with recurrent bleeding appears to be progressive, since this severe vasospasm was detected 48 hours after the final injection of blood. ") In our patient, the recurrent hemorrhage may have increased the severity and prolongation of vasospasm. 
Selection of the optimum therapy for such patients can be quite difficult. Surgical manipulation may prove harmful to the cerebral artery with vasospasm, and patients with recurrent hemorrhage have a poor prognosis. We found that topical application of papaverine solution was effective in reducing vasospasm in our patient during the craniotomy. An Ommaya CSF reservoir system allows topical application of vasodilating agents such as papaverine postoperatively. Segawa et $a l^{5)}$ evaluated papaverine injection at a relatively high concentration of $1-4 \%$ through a catheter placed in the cistern of patients with SAH. They reported transient oculomotor paresis and cerebral hemorrhage as side effects of papaverine injection. We used a more dilute papaverine solution $(0.1 \%)$, which had achieved a vasodilative effect during the craniotomy. Calcium antagonists also have vasodilative effects in the treatment of mild experimental vasospasm. ${ }^{3)}$ Oki et al. ${ }^{21}$ reported that cisternal injection of $2 \mathrm{mg}$ of nicardipine in patients after aneurysm clipping had no adverse effects. We administered a relatively low concentration of nicardipine through an Ommaya system in our patient. Postoperative angiography showed that these drugs were effective for treatment of vasospasm, but the development of cerebral infarction by the vasospasm was not completely prevented. We plan to use higher concentrations of these agents in the next similar patient.

We believe that the combination of topical application of papaverine and nicardipine with repair of the ruptured aneurysm and evacuation of subarachnoid clot will achieve a better clinical outcome for patients with localized but severe vasospasm and re- current hemorrhage.

\section{References}

1) Hunt WE, Hess RM: Surgical risk as related to time of intervention in the repair of intracranial aneurysms. $J$ Neurosurg 28: 14-20, 1968

2) Oki S, Kobayashi M, Yoshihara T, Yamada K, Sakoda K, Uozumi T: Effect of cisternal administration of nicardipine hydrochloride on cerebral vasospasm. Hiroshima J Med Sci 36: 75-79, 1987

3) Pasqualin A, Vollmer DG, Marron JA, Tsukahara T, Kassell NF, Torner JC: The effect of nicardipine on vasospasm in rabbit basilar artery after subarachnoid hemorrhage. Neurosurgery 29: 183-188, 1991

4) Saito I, Shigeno T, Aritake K, Tanishima T, Sano K: Vasospasm assessed by angiography and computerized tomography. J Neurosurg 51: 466-475, 1979

5) Segawa H, Saito I, Okada T, Nagayama I, Kitamura K, Takakura K, Sano K: Efficacy of intracisternal papaverine on symptomatic vasospasm. No Shinkei Geka 14: 847-854, 1986 (in Japanese)

6) Taneda M: Effect of early operation for ruptured aneurysms on prevention of delayed ischemic symptoms. J Neurosurg 57: 622-628, 1982

7) Zabramski J, Spetzler RF, Bonstelle C: Chronic cerebral vasospasm: Effect of volume and timing of hemorrhage in a canine model. Neurosurgery 18: 1-6, 1986

Address reprint requests to: K. Kataoka, M.D., Department of Neurosurgery, Kinki University School of Medicine, 377-2 Ohno-Higashi, Osaka-Sayama, Osaka 589, Japan. 